ESJ Humanities

\title{
Parenté à Plaisanterie ou Cousinage Entre les Dogons et les Songhays au Mali
}

\author{
Mahamar Attino, PhD \\ Enseignant - Chercheur, \\ à l'Institut de Pédagogie Universitaire (IPU), Bamako, Mali
}

Doi:10.19044/esj.2021.v17n13p1

Submitted: 09 October 2020

Accepted: 23 March 2021

Published: 30 April 2021
Copyright 2021 Author(s)

Under Creative Commons BY-NC-ND

4.0 OPEN ACCESS

Cite As:

Attino M. (2021). Parenté à Plaisanterie ou Cousinage Entre les Dogons et les Songhays au Mali. European Scientific Journal, ESJ, 17(13), 1.

https://doi.org/10.19044/esj.2021.v17n13p1

\section{Résumé}

Cette étude empirique est née de la sollicitude de la faitière de la culture Dogon (Ginna Dogon) qui a pour souci de vulgariser la culture Dogon tout en la situant par rapport aux cultures sœurs du Mali notamment dans ce papier celle particulière de leurs cousins Songhays. C'est dans ce cadre que les organisateurs de son $5^{\mathrm{e}}$ festival baptisé « Ogobagna », (signifiant l'écueil, tasse dans laquelle sont servis les repas) du Hogon, (chef traditionnel) de la communauté. A travers cette manifestation culturelle, les tenants voulaient faire la lumière sur les pans entiers de la culture Dogon dans un contexte particulier d'insécurité pluridimensionnelle (politique, économique, institutionnelle, sécuritaire, de rébellion délétère, de djihadisme, de terrorisme larvé voire de conflits communautaires). Bref dans un Mali où le vivre ensemble est de plus en plus remis en cause par ces temps qui courent. L'objectif principal de cette réflexion est d'éclairer la lanterne des lecteurs sur les contours la parenté à plaisanterie qui lie les communautés maliennes en général et celle des Songhays et des Dogons en particulier afin coudre le tissu social actuel amoché sinon déchiré. Pour mener à bien cette étude, nous avons utilisé un guide d'entretien en vue de collecter les informations qualitatives. Au bout du compte, les résultats auxquels nous sommes parvenu sont entre autres : la genèse du cousinage entre les communautés Dogons et Songhay a été présentée; puis les mécanismes de la parenté à plaisanterie ont été indiqués. Par ailleurs, une typologie du cousinage a été dressée. Les avantages 
et limites de la parenté à plaisanterie ont été définis. In fine le rôle du cousinage dans la résolution des conflits a été indiqué.

Mots-cles: Parenté à plaisanterie, cousinage, Dogon, Songhay, Mali

\title{
Joking or Cousin Relationship between the Dogons and the Songhays in Mali
}

\author{
Mahamar Attino, PhD \\ Teacher - Researcher at the Institute of University Pedagogy (IPU), \\ Bamako, Mali
}

\begin{abstract}
This empirical study was born from the solicitude of the leader of Dogon culture who seeks to popularize its culture while placing it in relation to others cultures of Mali, especially that of their Songhay cousins as seen as a case study in this paper. It is in this context that the organizers of its $5^{\text {th }}$ festival called it "Ogobagna" (meaning the reef, a cup in which the meals of the Hogon, traditional leader of the community are served). Through this cultural event, the supporters wanted to shed light on the entire sections of Dogon culture in a particular context of multidimensional insecurity (political, economic, institutional, security, deleterious rebellion, jihadism, latent terrorism or even community conflicts). This paper focuses on enlightening the readers on the outlines of jacking party which binds the Malian communities in general and that of the Songhays and Dogons in particular. This is done in order to stitch together the current social fabric that has been damaged or destroyed. To carry out this study, we used an interview guide to collect qualitative data information. At the end of the study, the results show the genesis of the cousinhood between the Dogon and the Songhay communities; the mechanisms of joking kinship were indicated; and the advantages and limits of joking kinship were defined. Ultimately, the role of cousinship in conflict resolution was also indicated.
\end{abstract}

Keywords: Cousinhood, joking kinship, Dogon people, Songhay people, Mali

\section{Introduction}

C'est la Commission d'organisation du cinquième Ogobagna (organisé par Ginna Dogon) qui a démarché une équipe pluridisciplinaire d'enseignants-chercheurs basée dans les structures de l'Université pour produire des documents sur les valeurs sociétales de leur communauté. Les commanditaires de cette étude voulaient aussi appréhender l'interaction entre 
les différentes cultures, dans un contexte particulier au Mali teinté de conflits communautaires ou du moins de remise en cause avérée du vivre ensemble. Pour l'histoire, cette 5 e édition du festival Ogobagna a eu lieu du 27Janvier au 2 Février 2020 à Bamako. Ce festival demeure surtout un instrument de dépassement de conflit par le biais de l'utilisation d'outils sociologiques (Indépendant, No 490 du Vendredi 7 Février 2020).

Dans un contexte particulier de questionnements, de crise identitaire adossée à une crise multidimensionnelle(sécuritaire, politique, de gouvernance, identitaire, et spirituelle),la requête de la communauté dogon trouve tout son sens dans la mesure où c'est l'unité nationale même dans son essence qui est en danger d'effritement .La noblesse de cet acte vient aussi du fait qu'à notre sens, cette idée de revisiter l'histoire de la culture ou l'histoire de la cohabitation des communautés vient du peuple qui à notre connaissance est le «plus ancien, le plus original du pays ».

Dans cette production nous allons nous évertuer à définir le concept de cousinage à plaisanterie en étudiant ses origines, ses manifestations ou mécanismes dans une première entrée.

Puis dans une seconde entrée, nous envisageons de faire la typologie du cousinage, tout en indiquant ses avantages et ses limites.

Et in fine, nous nous appesantirons sur le rôle du baromètre social que constitue le cousinage dans la résolution des tensions sociales dans un pays en quête de ses repères existentiels d'antan.

\section{Contexte et justification de l'étude}

La parenté à plaisanterie peut se définir comme une pratique sociale typique de certaines contrées africaines notamment en Afrique de l'Ouest et en Afrique centrale qui autorise voire parfois oblige les membres d'une même famille, certains noms de famille; certaines ethnies ou des habitants de la même région, territoire et provinces à se moquer ou à s'insulter sans risque conséquences aucune. Recevoir et donner des coups est le leitmotiv du cousinage. La parenté à plaisanterie autrement appelé «joking relationship » est un phénomène social collectif au Mali.En effet le cousinage permet le dialogue interculturel. Il consolide la cohésion sociale.

Quant à la parenté, elle est une relation sociale privilégiée, consanguine ou non, fondée sur l'existence réelle ou supposée d'une filiation commune, d'une alliance ou sur une adoption (Lévi-Strauss, 1949).

Aussi il convient de signaler que les liens de parenté s'articulent autour de la filiation, de l'alliance ou de la germanité. De façon générale la parenté s'exprime par la consanguinité (biologique) ou par la fraternité symbolique (amitié). La parenté à plaisanterie entre les communautés Dogon et Songhay relève de ce deuxième ordre. 
La cinquième édition du festival Ogobagna s'est tenue dans un contexte politique marqué par l'insécurité et la montée des antagonismes et clivages intercommunautaires. La communauté Dogon est originaire du centre du pays qui est un vieux bastion de la religion musulmane avec des références qui remontent à Sékou Amadou Barry, fondateur de la Dina du Macina (autrement appelée la Dina de Hamdallaye), puis du djihad Omarien qui s'était épanouit dans cette même région géographique (Grottes de Dinguimbere où Elhadj Omar Tall aurait trouvé la morten 18 ...).

C'est donc dans ce contexte particulier, d'interrogations, de suspicions, de méfiance entre les diverses communautés qu'intervient cette réflexion. Un contexte de menaces sérieuses de partition du pays, résultat d'un irrédentisme touareg répétitif et interrogateur surtout avec la menace « supposée » ou « réelle » de la création du fantomatique et hypothétique «Etat $\gg$ de l'Azawad, qu'intervient cette production.

Au même moment où le Nord et le Centre connaissent une implosion, le sud du pays, siège du Gouvernement central du Mali, il n'est pas de tout répits également : un fort tiraillement a lieu entre les formations politiques sur la gouvernance et ses déviances (corruption et de laxisme avéré) qui heurtent la conscience des citoyens ordinaires. C'est donc dans cette mouvance temporelle que la communauté Dogon à travers ce festival a voulu rappeler aux autres communautés ce qui nous unit, car comme le dit l'adage « la culture est ce qui reste lorsqu'on a tout oublié ».

En effet la communauté Dogon a, par le biais de sa culture, dit aux autres communautés du même pays, "nous ne sommes pas des montres, ni des athées », mais simplement des maliens comme vous, adorant les mêmes dieux, même si notre singularité est et demeure notre attachement marqué à nos coutumes, us et traditions. Les communautés Dogon ont voulu dire aux autres qu'ils sont un peuple pacifique, ayant toujours vécu en parfaite symbiose avec les communautés de voisinage, à travers l'histoire, aussi qu'ils sont et restent ce peuple spécifique admiré dont la culture a été vulgarisée par les anthropologues (Marcel Griaule), les hommes de science (Dieterlen,G,), et tant d'admirateurs venant du Mali ,mais aussi du monde entier. Les Dogons sont connus au Mali pour leur bravoure, pacifisme et honnêteté.

\section{Problématique}

En accédant à l'Independence, nombre de pays africains avaient leurs frontières artificiellement tracées au compas et à la règle par le colonisateur au cours de la fameuse Conférence de Berlin (1884-1885). Inutile de dire que ces frontières ont divisé des peuples aux identités similaires qui se sont trouvés du jour au lendemain dans des pays différents. La notion de nation est donc à bâtir pour la plupart de ces nouvelles créations étatiques. La nation se construit avec des peuples ayant vécu une histoire commune, mais aussi avec 
l'expression d'une volonté affichée de vivre ensemble. Les pères des indépendances ont utilisé comme stratagèmes pour renforcer l'unité nationale, la promotion des mariages mixtes et de la parenté à plaisanterie pour cimenter la cohésion sociale.

Le second paramètre à intégrer dans cette problématique est le renforcement de la construction nationale, ciment du développement du pays. L'unité nationale est un crédo qu'il convient de réussir car c'est après lui que suivra le développement. La première République du Mali l'a réussi en développant une politique du syncrétisme sur le plan administratif, en ce sens que les fonctionnaires originaires des régions du Nord sont automatiquement affectés dans les zones du Sud, et vice versa. Il en est de même pour les jeunes recrues dans l'armée, qui après la formation commune de base font obligatoirement un séjour calendaire dans les régions du septentrion étant les plus hostiles, les plus vastes, et les plus dangereuses, car les frontières demeurent floues et distantes. L'idée était que le séjour prolongé de ces citoyens de divers horizons fera qu'ils vont s'acclimater et se fondre dans la population hôte, aboutissant progressivement à une intégration, une fusion qui devient le dissolvant des différences ethniques et claniques. Une analyse à postériori de cette pratique met en exergue un bilan élogieux surtout qu'à notre humble avis le mérite est de l'avoir tenté.

Le Mali est un pays multiethnique, mais aussi multiculturel dans lequel chaque communauté est jalouse de ses spécificités ; même si force est de reconnaître que l'interpénétration par le biais du mariage mixte ou exogamique gagne de plus en plus le terrain surtout avec les générations actuelles.

Une autre particularité du Mali c'est d'avoir été le berceau de plusieurs formations étatiques à travers l'histoire : du Wagadu au Songhay en passant par le Mandé. Rappelons qu'après la dislocation de ces hégémonies, ces formations furent remplacées par des royaumes à base ethnique et /ou religieuse. Les ciments de la cohésion sociale dans ces entités, en dehors de l'appartenance ethnique et religieuse sont le cousinage et le recours aux dépositaires des traditions en occurrence les griots et hommes de caste.

Le cousinage est un pacte social scellé et inviolable qui lie deux clans à l'intérieur de la même communauté (Traoré et Diarra ; Maïga et Touré) ou entre deux groupes ethniques (Dogon et Songhay, Songhay et Mossi ; Bozo et Dogon). Il a généralement un fondement idéologique et remonte dans l'histoire des communautés. Il peut être aussi récent et volontaire (Maïga et Coulibaly).

Malgré son caractère à dominance qualitative, cette production notonsle est à dominance qualitative et a aussi puisé dans l'analyse du contenu des documents ethnologiques, anthropologiques, sociologiques et culturels sur les communautés Dogon et Songhay. 
Voilà un certain nombre de questions qui nous guiderons dans l'analyse de la tourmente au centre de notre pays, le Mali, même si l'objet de cet écrit reste centré sur l'analyse de la parenté à plaisanterie entre les communautés Dogon et Songhay.

Pour réussir cette étude, nous avons élaboré des questions de recherche, fixé des objectifs, formulé des hypothèses et avons élaboré une démarche méthodologique indiquée pour mieux cerner les contours de cette réflexion.

\section{Questions de recherche}

Nous avons aussi élaboré des questions de recherche pour vérifier la faisabilité de nos paramètres de recherche.

\section{Question principale}

Le cousinage à plaisanterie entre les Dogons et les Songhays peut-il être un moyen de renforcer la cohésion sociale dans un Mali en quête de son devenir?

\section{Questions spécifiques}

à quand remonte le cousinage entre les Dogons et les Songhays ?

Quels sont les types de cousinage chez les Dogons et les Songhays?

Quels sont les avantages et les limites du cousinage entre ces deux communautés?

en quoi le cousinage peut -être un vecteur traditionnel de résolution de conflit social ?

\section{Objectif principal}

L'objectif principal de cette réflexion est d'étudier l'origine, les manifestations ou les mécanismes de la parenté à plaisanterie (cousinage) chez les communautés Dogon et Songhay.

\section{Objectifs spécifiques}

étudier l'origine, les manifestations ou mécanismes de la parenté à plaisanterie chez les Dogons et les Songhays ; dresser la typologie du cousinage ; préciser ses avantages et ses limites ; indiquer le rôle du cousinage dans la résolution des tensions sociales.

\section{Hypothèses}

\section{Hypothèse principale}

La parenté à plaisanterie entre les Dogons et les Songhays est une résultante de l'histoire des deux communautés. 


\section{Hypothèses spécifiques} l'histoire ;

les liens de cousinage entre les Dogons et les Songhays remontent dans

la perpétuation du cousinage entre ces deux communautés a des avantages mais aussi des limites ;

la persistance de cette parenté à plaisanterie tire sa force dans la croyance des deux communautés à son caractère sacré et inaltérable ;

la parenté à plaisanterie entre les Dogons et les Songhays a résisté à toutes les turbulences du temps et est cité en exemple comme repère traditionnel de résolution de conflit.

\section{Démarche Méthodologique}

Dans un premier temps nous avons défini notre champ d'étude qui porte sur les aires culturelles des communautés Dogon et Songhay. Dans un second temps nous avons indiqué notre échantillonnage qui a porté sur le témoignage et les récits de scène de vie de quelques personnes ressources mais aussi de l'analyse du contenu des discours de nos répondants. Enfin nous indiquons la méthode d'analyse utilisée pour réussir cette production scientifique.

Pour mener à bien cette étude empirique, nous avons usé de l'entretien avec des personnes ressources. C'est donc une étude que nous avons voulu strictement qualitative. Cette analyse qualitative d'orientation socio anthropologique s'est appuyée sur la transcription et l'interprétation des discours recueillis auprès de différentes personnes ressources rencontrées sur le terrain. Nous avons donc procédé à des entretiens semi-dirigés puis à l'analyse du contenu des discours.

Pour la mener à bien, nous avons aussi élaboré des questions de recherche, fixé des objectifs et formulé es hypothèses pour mieux cerner les contours de cette réflexion.

\section{Principaux résultats}

4.1. Le cousinage : genèse du concept.

Le cousinage à plaisanterie peut se définir comme un baromètre social à travers lequel les communautés ou les clans et les individus peuvent s'invectiver, se saboter tout en s'éduquant dans la mesure où ils se servent de lien sociétal pour redresser les torts sans pour autant nuire à autrui.

En effet la vie en communauté rime avec des rixes, des mésententes qui, si elles ne sont pas gérées avec tact et parcimonie, peuvent aboutir à des conflits pouvant mettre en mal le vivre ensemble. En plus de l'action des dépositaires de la tradition comme les griots et les hommes de caste, dont la mission est de coudre le tissu social, en faisant admettre à chacun son tort sans peine d'être rejeté ou contredit. Nos ancêtres ont institué le cousinage qui est 
le dernier recours pour résoudre définitivement les litiges intracommunautaires et intercommunautaires.

Le cousinage est une arme dont dispose les sociétés claniques pour esquiver la débâcle sinon la déconfiture de la société. A travers Ogobagna, les Dogons «veulent se servir de la culture pour lutter contre l'implosion sociale » dixit l'animateur vedette de l'événement.

Comme indiqué plus haut, le cousinage a été créé par les ancêtres dans le but de cimenter les liens communautaires non seulement pour les temps passés mais également pour les temps présents mais aussi ceux à venir. C'est la raison pour laquelle la plupart de ces cousinages sont gravés dans le marbre par des pactes soit de sang ou de parole d'honneur inviolable sous peine de discrédit et de bannissement.

La profondeur de l'univers du cousinage est à chercher selon les anthropologues dans les relations entre alliés et consanguins, raison pour laquelle ils parlent de liens avunculaires (Marion, 2008 ; Lefèbvre, 1985).

Pour une meilleure compréhension de la parenté à plaisanterie entre les communautés Songhay et Dogon, une présentation de certaines valeurs culturelles ou de certains référents culturels et même cultuels s'avère nécessaire, car pour mieux connaitre un peuple, il faut interroger son âme, ses croyances, ses rites, ses us et coutumes surtout que ces deux peuples dont il est question ont en commun d'avoir été très animistes et conservateurs, même si les Songhay en apparence ont tourné le dos aux dieux des ancêtres des suites d'une islamisation très ou trop poussée. Dans le passé, la mythologie ou la cosmogonie Songhay tournait autour d'un polythéisme avéré qui a beaucoup de similitude avec celle des Dogons. Pour schématiser, on peut dire qu'au sommet de la hiérarchie des forces de l'univers, le peuple Songhay croit à l'existence d'un démiurge (N'Debi) ; puis à celle plus lointaine de Ir-Koï (notre maître). Au total, notons que les Songhays croient à une multitude de choses en nombre pair dont principalement :

l'être et son double ;

le Sonianké et sa chaine ;

le tierko et son œuf;

le Sorko et son «Torou »;

le Zima et les Holé (Baulnois, H. Boubou, Empire de Gao ..., P 22).

La signification anthropologique de ces concepts titille le polythéisme de cette communauté : la dualité ou le dualisme caractérise la croyance Songhay. En effet il y a presque toujours un binôme dans les pratiques Songhay : tout être humain a son double, son sosie, ou en terme anthropologique son «ombre», sa silhouette. L'élément de la deuxième croyance porte sur le magicien et sa chaine. Le troisième fondement de la religion songhay est le sorcier et son œuf. Le quatrième pilier de cette croyance 
est le pêcheur et son idole. Enfin le dernier élément de cette idéologie porte sur le maître des possédés et le panthéon des dieux songhay notamment Dongodachi (dieu de la foudre), Marou tichirey (dieu de la pluie et de la prospérité), Bagembeza (dieu de la guerre), etc qui peuplent l'olympe du Songhay ganda (terre ancestrale des Songhays).

L'aire géographique où vivent les Songhays est très peu différente de celle des Zerma dont l'habitat traditionnel s'étend de Hombori, Djenné (Mali) à Gaya (Niger) et à la région de Kandi (haut-Dahomey). Il y a également de forts groupements de Songhay dans le Nord du Burkina (cercle de Dori) à Wanobia (Cercle de Gaya) et dans le Yatenga (Nord Burkina-Faso (Boubou, 1967, P 9).

L'influence linguistique des Songhays et des Zerma existe chez les Gourma. Il s'agit notamment 'un langage secret des circoncis,( idem , P9).les données sociologiques indiquent que les Songhay pratiquaient le matriarcat(Tierko) et le patriarcat (Sonianké).Les Songhay ont une secte absolument hermétique, celle des Tierko ( H. Boubou, 1967, P 10).Du fait du matriarcat ,chez les Songhay, le pouvoir se transmet d'office par le lait de la mère (Le neveu succède au roi).Les Songhay ont le pouvoir de lévitation (pouvoir de voler),les Tierko et les Soniankés émettent par tous les orifices de leur corps une lumière éblouissante provenant de l'élément moteur qui leur permet de voler (Boubou, 1967, p.15). Les Songhays ont aussi le pouvoir de saisir le double d'une personne, d'un animal (idem, P15).

Quant aux Dogons, ils occupent le plateau, comme aire géographique du même nom. Ce plateau est dressé au centre du pays qui surplombe une vaste plaine appelée Plaine du Seno-Gondo. Notons que le plateau Dogon se prolonge au Burkina -Faso par les contreforts du plateau de Banfora. Le milieu géographique où vivent les Dogons est un univers peuplé de falaises et de plaines, une zone de production agricole sans pareil avant le cycle infernal des sécheresses répétitives des années 1973,1984 et 2012 et les effets pervers du changement climatique. Les Dogons sont un peuple laborieux et discipliné qui adorent les forces de la nature avec laquelle ils vivent en parfaite symbiose. Des chercheurs pensent avoir trouvé les données élaborées d'une philosophie africaine savante du monde chez les Dogons de la falaise de Bandiagara (Griaule, P 11).

Les Dogons sont un peuple très attaché à la religion traditionnelle, celle des ancêtres qui implique la vie en harmonie avec la nature teintée d'interdits à ne pas braver et à respecter scrupuleusement. C'est un peuple d'agriculteurs et de guerriers.

Il est à noter que l'origine du cousinage remonterait dans l'Antiquité le long de la vallée du Nil où s'est épanoui la gigantesque et brillante civilisation pharaonique des suites de l'assèchement du Sahara au III e millénaire. Quant à la genèse du cousinage entre Songhays et Dogons, elle 
remonte à l'apogée de l'Empire Songhay. En effet, il a été rapporté dans le Tarik el Fettach que l'empereur Songhay en occurrence Sonni Ali Ber, au cours de ses multiples expéditions et razzias contre les royaumes du Yatenga et celui des Gourmantchés, avait l'habitude de faire escale au pays Dogon et plaisantait beaucoup avec les gens pour éviter la fuite des habitants des différents villages à chacun de ses passages. Sonni Ali aurait fait comprendre aux Dogons qu'eux comme lui sont issus d'un peuple animiste très attaché à sa culture et est - non belliqueux fait qu'il signa avec eux un pacte de nonagression qui le lie lui aussi bien que toute sa descendance. Selon le même pacte jamais un songhay ne ferait du mal ou un tort quelconque à un Dogon. Ce pacte a été respecté du vivant de Sonni le grand (1464 à 1490, période de son règne) à aujourd'hui. Sonni Ali aurait demandé aux Dogons ne plus jamais s'en fuir à sa vue dans la mesure où, il ne fait que traverser leurs terres pour arriver à celles de ses rivaux, en pays Gourmantché (Mampoursi) et Mossi (Yatenga).

Notons au passage que le cousinage entre Songhays et Dogons avec le temps, fut étendu à toutes les communautés du Nord, notamment les communautés Touarègues et Arabes.Il convient d'ajouter que le cousinage avec les autres communautés du Nord du Mali fut axé sur un pacte de promesse réciproque d'échanges des céréales comme le mil et le sorgho, produits par les Dogons contre le sel gemme, les dattes et les étoffes venant du Nord. C'est la sauvegarde de cette entente économique qui serait à la base de du cousinage à plaisanterie entre les communautés Arabe, Touareg, et Dogon.

Quant au cousinage entre Dogon et Bozo, le dépositaire traditionnel nous a révélé que ce qui est à l'origine du pacte sacré entre les deux communautés n'est pas dû au à la légende couramment admise du pacte de sang au cours de la famine de l'un des frères (où le Dogon aurait coupé une partie de sa cuisse pour calmer la faim du Bozo à son insu). L'informateur nous fit observer que dans nos traditions, «Kalifa» est difficile: on a l'obligation morale de s'occuper bien de l'enfant d'autrui confié à soi. Et selon lui, c'est qui caractérise l'histoire du petit Dogon et du petit Bozo. Le fil du récit c'est qu'un Dogon devant aller en aventure, aurait confié son enfant à un Bozo qui avait un enfant du même âge que celui du Dogon. Après des années de vie, les deux enfants sont tous décédés. A son retour le Dogon alla chez le Bozo pour récupérer l'enfant à lui confié. Le Bozo fit comprendre au Dogon ce qui arriva. Le Dogon lui dit allons au cimetière, formuler des prières pour le repos des âmes des défunts enfants. Arrivés au cimetière ils trouvent les deux enfants en train de s'amuser. Le Bozo demanda au Dogon, «tu m'as trompé ? » ou «tu t'es foutu de moi ! » Non répondit le Dogon «Kalifa ka guelèn » (signifiant littéralement : la chose prêtée est inviolable). Ils prirent les enfants et formulèrent une prière en buvant de la même eau, en ces termes : 
puisse dieu ne point salir notre sang ! Ce récit pathétique plein de renseignements et de sagesse. C'est le lieu de spécifier qu'entre les Dogons et les Bozo il existe ce que les ethnologues appellent un totémisme sexuel (ils ne s'unissent ni par le mariage ni par des rapports sexuels). Mieux, ils n'assistent même pas aux funérailles des uns et des autres, tellement qu'ils se sentent un.

\subsection{Manifestations ou mécanismes du cousinage à plaisanterie}

Le cousinage s'effectue à travers des mécanismes comme la plaisanterie, les proverbes, les anecdotes comiques, le changement de cap, les échanges de plats de haricot, etc.

$\mathrm{O}, \mathrm{P}$, une personne ressource nous a rappelé le dicton bambara selon lequel «Mogo don,so don,yerè don dé gnogon té ». Comme quoi il ne sert à rien d'être un expert en zootechnie ou en humanisme, que la meilleure des connaissances est celle de se connaître soi-même ; le « connais-toi toi-même » de Socrate.

Selon O. P, le « cousinage signifie s'apprécier, s'accepter et cela même dans la divergence ». Ceci renforce la connaissance de soi et des autres, car on s'auto -protège par l'intermédiaire du cousinage. Ainsi dans la pratique, quel que soit le problème, on s'accepte, on s'écoute, on laisse ou on recule (cède) quand le cousin intervient. Mais dans cette démarche, on doit être juste et équitable, on ne doit pas abuser. Selon O.P, le cousinage entre songhay et Dogon signifie respect réciproque, dambé (dignité). D'après, O.P, le cousinage entre Dogon et Songhay tire ses racines du fait que les songhays et les Dogons seraient à l'origine des frères. Il base son argumentaire sur le fait que les Dogons se seraient confiés à leurs ainés les Songhays. D'après lui, les Mandinka (Keita) sont les enfants des Soninkés. De même que les Songhay (Maiga, Touré), sont aussi les enfants puinés des Sarakhollés. Donc les Keita et les Maiga (patronyme ayant comme par enchantement la même signification: Maiga signifie le roi, le maître) sont les ainés et les Dogons (Dogono) seraient les cadets.

Cette personne très imbue de la culture malienne, a rapporté que le Mali serait issu de la combinaison de «trois personnes : Soninké, Songhay, et Dogon ». Aussi il fit observer que les Dogons sont les descendants de la vieille femme Soninké mariée au diable ; ce qui corrobore l'origine extra- terrestre des Dogons véhiculés par les légendes populaires de leur terroir.

Quant à l'origine des Dogons, le narrateur rappela que les Dogons, selon une chanson populaire dogon, seraient venus du Mandé. Mais il s'empressa de dire qu'en fait les Dogons primitivement seraient venus du Wagadu, et auraient plutôt transité par le Mandé avant de s'installer définitivement dans le plateau du même nom. La question reste de savoir s'ils sont venus trouver sur place les Tellems (noyau primaire du peuplement du Mali) ou bien selon l'hypothèse que nous formulons : les Dogons font partie 
du premier peuplement du Mali, et les vestiges, les grottes et abris souterrains Tellems en sont les témoignages les plus éloquents. Même si à notre humble avis ce sont les Mandinka et sous- clans qui sont issus de ce peuple plusieurs fois séculaires et foncièrement attachés à leurs us et coutumes, ce qui constitue d'ailleurs aujourd'hui un terreau propice à l'amalgame : «tu es Dogon, tu es animiste » que brandissent les djihadistes et acolytes. L'autre revers de la médaille existe : «tu es peul, donc tu es djihadiste ». Ces crédos ont cours aujourd'hui dans un contexte sociologique, et politico économique difficile du pays. Il reste vrai, que la primeur reste aux pensées de nos répondants !

B.O. le Hogon (chef traditionnel) au cours du festival nous a surtout entretenus des mythes et de la cosmogonie Dogon. En effet selon lui «dieu a existé chez les Dogons avant la religion. En ce sens que le dieu Amma, est matériel et immatériel : tout ce qui est autour de l'homme, est dieu notamment l'air, le vent, l'eau. Pour atteindre dieu, selon les Dogons, on n'a pas besoin d'intercesseur. Ainsi une pierre, un arbre, une mare sont les signes patents de son 'omniscience et de son omnipotence ». Les Dogons restent les derniers remparts contre la dénaturalisation. Ils n'ont pas tout laissé, ils résistent culturellement en s'attachant aux valeurs ancestrales, ce qui fait qu'on les accuse à tort d'être des mécréants sinon des athées tout courts. Ce qui fait d'eux des victimes expiatoires toutes désignées pour des pseudos défenseurs de l'Islam en occurrence les Djihadistes Koufistes ou Iyadistes et les autres zébrures d'Alkayida.

Selon le Hogon du festival Ogobagna, la cosmogonie Dogon enseigne qu'à la fin des temps, il y aura de sérieuses contradictions qui sont les signes avant- coureurs de l'apocalypse. Par exemple «nos créatures nous tuent au lieu de nous être utiles. Le châtiment n'est plus individuel, mais collectif » dans le sens où la peine n'est plus personnelle car la punition devient collective comme aux temps bibliques : Sodome et Gomorrhe. Ce qui fait que les Dogons ont eu la prémunition et «n'ont pas tout jeter, » dans le sens du conservatisme primitif athée.

B .0, précise aussi que selon la cosmogonie Dogon : «la beauté n'est pas physique, et qu'elle réside dans l'âme et non dans le cœur ». Pour illustrer cette assertion il développa «l'anecdote de la calebasse neuve laissée au bord d'une route carrefour ». Lorsque tu dépasses un tel ustensile, tu te dis sûrement que plusieurs personnes l'ont vu avant toi. Tu hésites, tu le laisses. Tu te convaincs de partir ou bien tu te mets dans un angle mort pour observer. Le premier à aller avec la calebasse «va aller avec le malheur et le bonheur lié à ladite calebasse ».

La symbolique de la calebasse représente la femme chez les Dogons. Selon les Dogons, c'est dans la maison qu'on vérifie si la femme est bonne ou pas, car l'intérieur peut ne pas être comme l'extérieur. Selon le peuple Dogon, «nous sommes des créatures de dieu » alors que l'Islam enseigne que « nous 
sommes des esclaves de dieu, » et le christianisme, quant à lui, soutient que nous sommes «fils » de dieu. Ceci corrobore la théorie selon laquelle les Dogons seraient un peuple extra- terrestre (fruit de l'union entre une déesse et un Soninké).

\subsection{Typologie du cousinage}

Il y a deux types de cousinage : celui qui est entre les clans d'un même groupe ethnique qu'on va appeler le cousinage de type vertical. Et le cousinage entretenu entre des ethnies différentes, on va le nommer cousinage horizontal. $\mathrm{Si}$ le cousinage vertical renferme les liens claniques, celui dit horizontal revigore les liens sociaux et réglemente le vivre ensemble à l'échelle d'une nation ou au-delà ; et cela malgré les affres de la balkanisation imposée par le fait colonial.

La parenté à plaisanterie qui lie Dogons et Songhay relève du type horizontal et peut- être d'une grande utilité dans un contexte trouble comme celui que vit le Mali tout entier de nos jours. Le Mali rappelons- le est une mosaïque de 13 ethnies qui non seulement ont vécu ensemble pendant des millénaires, mais aussi ont pu tisser des liens de sang et entretenu des liens de cousinage et de ce fait, ont pu endiguer beaucoup de chocs et de remous sociaux et culturels. Les exemples ne finissent pas: entre Senoufo et Minianka ; Bwa et Peuls ; entre Malinké et Soninké, etc.

Les Etats multi ethniques ont intérêt à cultiver ces valeurs sociétales séculaires pour booster un climat de confiance réciproque, de tolérance, et d'entente mutuelle afin de renforcer le vivre ensemble secoué par les soubresauts du séparatisme et les amalgames du néo prosélytisme importé et à nous imposer.

\subsection{La parenté à plaisanterie : avantages et limites}

Les sociétés humaines inventent des paradigmes comiques, des règles de conduite, collectent, édictent des lois à ne pas transgresser, et des normes qui s'imposent à tous. Le premier mérite de la parenté à plaisanterie est de mettre le doigt sur les tares sociales par l'intermédiaire du comique : ne dit-on pas que le rire soigne?

Le cousinage permet d'assener des vérités sans vexer ; de rectifier ou de corriger des erreurs sans mettre l'autre dans l'embarras. Il permet de faire des observations pertinentes pour rectifier des erreurs souvent grossières surtout quand elles viennent de personnes haut perchées dans la hiérarchie sociale (entre un président et un citoyen lambda). A la limite il permet d'éduquer, d'orienter, de sensibiliser, d'attirer l'attention sur de faits importants (une personne qui s'adresse à son chien et l'appelle par le nom de son cousin ; ou quand un médecin Dogon dit à son patient va mettre cette capote sur ton « Maigakeni » etc.). Le sens anthropologique de ces expressions 
c'est de dire que les cousins sont autorisés à se « chosifier» sans aucune offense. Les vertus du cousinage sont illimitées et permettent d'harmoniser la vie en société dans le respect mutuel. Les vertus du cousinage sont éducatives, sécuritaires et thérapeutiques, car elles permettent de normer la société tout en l'harmonisant. Toutes les classes d'âge, hommes, femmes, jeunes et vieux s'adonnent à cette pratique sans discrimination aucune.

Le cousinage est le moteur de la rupture de l'endogamie et de l'ouverture progressive à l'exogamie. A titre illustratif, les songhays sont connus pour leur réticence à la pratique de l'exogamie, mais paradoxalement ils acceptent malgré tout de donner leur fille en mariage « aux plus vilains » en occurrence es Dogons. La raison est à chercher dans la quête de la pureté, de la noblesse, du sens élevé de la dignité constatée chez ce peuple. Ce qui disculpe les Songhays très souvent taxés de xénophobes, de régionalistes sinon d'ethnocentristes tout court.

Les limites que nous connaissons à cette pratique sociale sont l'âge, dans la mesure où au Mali nous vivons dans une société gérontocratique où le droit d'ainesse est une loi invisible (non écrite) mais respectée par tous. Une autre entrave ou limite est l'interdiction totale d'injures et dire des grossièretés (les cousins à plaisanterie peuvent se dire tout sauf s'insulter réciproquement les parents).

Le cousinage n'autorise pas également de saboter ou de s'attaquer à la religion des uns et des autres, la laïcité étant un credo défendu mordicus par tout le Mali, surtout en cette période de charia «proposée » ou en voie de l'être. Mais, il est autorisé de faire des sarcasmes relatifs aux religions de temps en temps avec un esprit tout à fait fairplay et sans trop de méchanceté. Enfin dans le cousinage il y a aussi l'obligation de la réciprocité. Quel que soit le courroux, le cousinage peut désamorcer la bombe prête à éclater, car les parties en litige se rendant compte qu'ils sont des cousins à plaisanterie, se disent des méchancetés tragicomiques et cèdent la plupart du temps. Le cousinage n'admet pas non plus le mensonge et la délation, ni n'autorise le vol et le vice.

\subsection{Rôle du cousinage dans la résolution des tensions sociales}

La société malienne a érigé des gardes- fous afin de réguler les rapports entre les individus et ceux entre les différentes communautés.

Généralement dans nos sociétés on sert des personnes âgées, des hommes du culte (marabouts et prêtres), des griots /hommes de castes et souvent des classes d'âge pour désamorcer les tensions sociales. Lorsque tous ces « démineurs » potentiels échouent, le médiateur ultime reste la pratique du cousinage à plaisanterie à laquelle aucun malien digne de ce nom n'échappe ou ne saurait se soustraire. 
En effet au Mali, le «sanankouya » est constitué d'une myriade de règles non écrites, mais qui sont strictement respectées par tous les membres des communautés liées par cette parenté. Le sanankouya tire ses racines de l'histoire, et comme le dirait l'autre « un peuple sans histoire, est un peuple sans âme ». L'alliance à plaisanterie permet la cohésion sociale. Elle est une richesse culturelle essentielle du Mali. Elle est un héritage culturel du Pays. On ne finirait pas de répertorier tous les avantages de cette pratique sociétale tellement ils sont nombreux, mais retenons entre autres : le cousinage permet d'apaiser les tensions dans les familles, entre différents clans de la société ; à travers les amusements, les rires et les satires de cimenter la cohésion sociale ; il permet et entretient le dialogue interculturel, etc.

Comme l'a assené O.P parlant du peuple malien «parmi nous, il n'y a pas de mauvaises personnes. Mais par nos actes, on s'est accommodé avec les mauvaises personnes, ce qui déboucha sur des drames ». S'agissant des médiateurs internationaux, nationaux ou locaux, 0 . $\mathrm{P}$ poursuit pour dire : «celui qui lèche le revers et la paume de la main ne pourra jamais dire la vérité »; pour dire que ... la neutralité doit être de mise pour les médiateurs car on ne peut être «juge et parties ».

Que peut-être le rôle du cousinage dans la résolution de la crise actuelle que vit le Mali?

La crise au Mali a duré dans l'espace et dans le temps. En effet depuis 1990 jusqu'en 2020, on peut affirmer sans ambages que le pays dans son entièreté est secoué par une crise ethnique localisée au Nord avec l'irrédentisme Touareg. De locale la crise se mut en crise sécuritaire, identitaire et indépendantiste sinon sécessionniste avec la menace de création d'un nouvel Etat dénommé l'Azawad.

La crise s'étendra à presque toutes les régions du Mali avec comme épicentre actuellement Mopti. A partir de là, la crise se mua en crise religieuse doublée de crise identitaire et communautaire qui oppose principalement les communautés Dogon et Peules qui ont vécu sur le même espace géographique pendant des siècles. Ceci pose un problème de gouvernance, mais aussi de cohabitation entre deux religions bien ancrées dans le terroir : L'Islam et l'animisme qui sont entretenus par des préjugés comme celui énoncé ciaprès : « tu es peul, donc tu es djihadiste ; tu es Dogon, donc tu es animiste ».

Le cousinage à plaisanterie est une vertu cardinale qui implique humilité, respect et soumission. Si on voit que le conflit persiste c'est qu'il y a derrière des intérêts supranationaux, aussi il y a le fait que les cadres des communautés qui résident à Bamako, attisent le feu, la haine et la financent. L'Etat dans ses tentatives de résolution de la crise (ironie du sort) s'appuie sur ces mêmes cadres.

Passer par le biais du cousinage pour juguler la crise est une thérapie que l'Etat doit expérimenter, car les autres mécanismes ont montré leur limite. 
Pour résoudre le problème, il faut « libérer la parlerie » en acceptant de s'asseoir, de discuter, d'échanger en s'appuyant sur un plaidoyer axé sur le cousinage. Il faut organiser une table-ronde ou un «bavardage national » sur le vivre ensemble afin de crever l'abcès.

\section{Discussion des résultats}

Les résultats auxquels notre étude a abouti nous permettent de comprendre les liens entre les différents paramètres du problème de recherche étudié. En effet l'étude a permis de retenir qu'il y a des liens évidents ou patents entre les fondements culturels de la parenté à plaisanterie chez les peuples Songhay et Dogon. Aussi que les données analysables sont la reconnaissance d'un pacte sacré même s'il reste verbal scellé entre ces deux peuples depuis la nuit des temps. Le respect strict du dit pacte est l'instrument de perpétuation du cousinage entretenu de génération en génération .la sauvegarde des intérêts matériels et moraux des deux communautés est aussi un fait majeur qui donne sens à la pratique multiséculaire du cousinage entre les communautés Songhay et Dogon.

Notre étude étant strictement qualitative, nous avons procédé à la construction de typologies pour regrouper les variables qui vont ensemble. C'est aussi la raison pour laquelle nous avons fait une analyse du contenu des discours de nos répondants pour mieux saisir le sens ou les messages des textes ou des entretiens retranscrits. Cette étude squelettique d'un phénomène social total au contour circonscrit qui est la parenté à plaisanterie, nous a permis de nous rendre compte de toute la complexité des rapports sociaux constitutifs en matière de relation intra et intercommunautaire.

A travers cette interprétation, nous voulons montrer la pertinence ou la validité de nos résultats par rapport au problème de recherche, à nos questions de recherche, aux différentes hypothèses et à notre cadre de référence.

Plusieurs auteurs se sont intéressés à la thématique que nous abordons dans ce papier. Certains abordent la question de la même manière que nous même si le contexte et la géographie ne sont pas identiques (Kouadio Kouadio $\mathrm{Y}$,) aborde la question dans son analyse sur les « alliances inter-ethniques et parenté à plaisanterie ou dynamique d'une dédramatisation endogène des conflits socio-politiques : le cas de la Côte d-Ivoire » où il note avec netteté que les «affrontements verbaux entre cousins ne sont en réalité que des moyens de décrispation sociale ».

Également Djibril Tamsir (2005) insiste sur les initiatives de valorisation des capacités africaines endogènes dans la gouvernance et la gestion des conflits notamment par le biais de la parenté à plaisanterie.

Kaba (2015) définit la parenté à plaisanterie comme « des outrages et attaques que certaines personnes se plaisent à développer vis-à-vis d'autres 
personnes sans pour autant parvenir à un drame », ce qui indique le caractère thérapeutique de la pratique du cousinage.

L'alliance cathartique qui consiste à dire qu'il y a du Dogon dans tout Bozo ou Songhay dans tout Dogon traduit avec force le poids du cousinage chez les communautés citées.

La parenté à plaisanterie peut se définir comme une parenté fictive, métaphorique instituée entre groupes socioprofessionnels (castes), villages, régions, patronyme ou entre ethnies comme c'est le cas qui fait objet de notre réflexion.

Il est vrai que Alain Joseph (2002) fait observer que le cousinage put naître aussi de conflits au travers d'alliances guerrières comme entre les Mossis et les Samogo (peuls). Aussi que la parenté à plaisanterie peut lier des peuples aux modes de vie différents comme entre les Touareg et les Dogons, ou comme entre les Peuls et les Bwas.

Il y a lieu de préciser que le cousinage implique une «impolitesse rituelle » où les apparentés rivalisent d'ardeur et de créativité afin de trouver des insultes plus piquantes, drôles et comiques(actes de sottise, de gourmandise, d'alcoolisme, de non bravoure,etc).la parenté à plaisanterie ne connait presque pas de limites dans sa pratique car même les morts sont souvent titillés par leurs cousins : par exemple au cours de l'enterrement d'un grand syndicaliste Dogon au Mali, les fossoyeurs n'ayant pas pu bien dimensionner la tombe, un Songhay assistant au dit enterrement s'est exclamé à l'étonnement général de l' assistance : même mort, un tel(en nommant le défunt )continue de déranger !

Les railleries ethniques issues du cousinage des bienfaits certains pour la vie en communauté.

On peut noter une authenticité de nos résultats obtenus car ils sont conformes à nos questions de recherche posées au départ, aux hypothèses formulées, ce qui nous procure une satisfaction morale.

Nous constatons aussi qu'il n'y a pas d'écart entre les résultats attendus et les résultats atteints. Si ce n'était pas le cas les raisons de la divergence auraient été avancées.

Les sociétés coutumières africaines ont en commun d'être pyramidales même si l'histoire sinon la géographie peut introduire certaines nuances (explications ou écarts par les déterminants sociaux et celles parles logiques d'actions individuelles).

C'est le lieu de dire que notre étude est loin d'être exhaustive, elle a des limites certaines: le caractère brulant du sujet, le processus d'échantillonnage qui s'est focalisé sur les quelques personnes ressources acteurs du festival Ogobagna qui aurait été plus expressif s'il s'étendait au pays dogon dans son entièreté ;l'absence de données quantitatives qui auraient pu mieux illustrer certains faits; les manquements et erreurs qui 
auraient pu se glisser dans les traductions, la transcription des discours, entre autres.

\section{Conclusion}

La parenté à plaisanterie est une valeur sociétale de première importance au Mali. Elle est une institution traditionnelle qui tire ses racines de l'histoire et a pour objectif de consolider les rapports sociaux, de faciliter le vivre ensemble et de résoudre sans coups férir les antagonismes et rixes inhérents à la vie en communauté.

Le cousinage entre les Songhays et les Dogons est multiséculaire et est l'un des mieux entretenus et jalousement gardé et respecté par les deux communautés. Ce cousinage relève du type horizontal et est d'autant plus marqué que les communautés sœurs du Mali commencent à véhiculer l'idée selon laquelle il y a une grande complicité entre les tenants à ce cousinage, en ce sens qu'il frôle la complicité sinon le népotisme même dans l'administration entre les deux ethnies jusqu'à ce qu'il soit courant d'entendre dire « que partout où il y a des Songhays, ils appellent les Dogons auprès d'eux!».

Les résultats de cet article sur le cousinage à plaisanterie indiquent que :

la parenté à plaisanterie a des avantages et des inconvenants ; aussi que le cousinage à plaisanterie joue un rôle de régulateur, de baromètre social car il permet la résolution des conflits entre tenants (partenaires) et par là entretient la quiétude sociale ;

dans un contexte de crise multidimensionnelle qui est celui du pays aujourd'hui, les décideurs doivent y recourir afin de juguler les antagonismes béants qui opposent les communautés jadis sœurs et qui de nos jours se regardent en chien de faïence.

Cultiver la parenté à plaisanterie est une arme efficace de dédramatisation endogène des conflits dans des Etats pluriethnique comme le Mali. La parenté à plaisanterie reste donc une arme de nouvelle gouvernance aux mains des décideurs.

A travers cette étude nous sur la pratique de la parenté à plaisanterie entre les Dogons et les Songhays au Mali, nous avons trouvé que le cousinage est un puissant moyen de préservation de la stabilité sociale.

Le cousinage entretient la dynamique endogène de résolution des conflits car il agit comme une thérapie qui participe quotidiennement à la régulation sociale. Aussi que cette pratique sociale multiséculaire a des avantages certains pour les gouvernants et gouvernés.

Au bout du compte nous affirmons que toutes les hypothèses formulées en amont de cette étude ont été confirmées. Notre étude revisite la parenté à 
plaisanterie entre les communautés Songhay et Dogon dans le but de renforcer ses fondements et magnifier ses vertus pour un mieux vivre réussi au Mali. D'autres chercheurs pourront approfondir la réflexion en se basant sur des données quantitatives.

\section{References:}

1. Boubou, H. (1967). « L'Histoire traditionnelle d'un peuple. Les Zerma-Songhay », Présence Africaine, Paris.

2. Boulnois, J. \& Hama, B. (1954). «L'Empire de Gao. Histoire et magie des Sonrais », Paris Maisonneuve, Vol 1,182 p.

3. Lévi-Strauss, C. (1949). «LES structures élémentaires de la parenté », Paris-la Haye, Mouton.

4. Doquet, A. (1999). «Les masques dogons : de l'objet du Musée de l'Homme à l'homme objet de musée », Cahiers d'Etudes Africaines, N0, 155-156.

5. Dieterlen, G. (1999). «Les Dogons. Notion de Personne et mythe de création », Paris, Harmattan.

6. Djibril Tamsir, N. (2005). «La parenté à plaisanterie: origine, fonction préventive et régulatrice dans l'espace Ouest africain » in Initiatives de valorisation des capacités endogènes dans la gouvernance et la prévention des conflits, Tome 2.

7. Griaule, M. (1976). «Dieu d'eau », Paris Fayard,3 e édition, $222 \mathrm{p}$

8. Rouch, J. (2005). « Les Songhay », L'Harmattan, 100 P.

9. Jean Rouch (1997). «Hommes et les dieux du fleuve. Essai ethnographique sur la population Songhay du Moyen Niger »,284 p.

10. Jean Rouch (1960). «Religion des Songhay », Paris, PUF,325 p

11. Joseph Sissao, A. (2002). "Alliances et parenté à plaisanterie au Burkina-Faso »,Sankofa et Gurli Editions.

12. Kaba, M. (2015). «Les alliances et parentés à plaisanterie: une richesse culturelle en Afrique »

13. Kouadio Kouadio, Y. (2020). «Alliances inter-ethniques et parenté à plaisanterie ou dynamique d'une dédramatisation endogène des conflits socio-politiques : cas de la Côte d'Ivoire» in Actes du colloque international sur « royauté, chefferie traditionnelle et une nouvelle gouvernance », Editions Dagekof.

14. Kouyaté, S. (2003). «Le cousinage à plaisanterie: notre héritage commun », Ganndal,96 p.

15. Ki-Zerbo, J. (1972). « Histoire de l'Afrique noire d'hier à demain », Paris, Hatier.

16. Ricord, C. (1992). «L'eau chez les Dogons : peuplement, croyances, utilisations », Mémoire de DEA, Ethnologie, Université de Provence Aix-Marseille, 200 p. 
17. Zahan Dominique (1970). « Religion, Spiritualité et Pensée africaines », Paris ; Payot ; $244 \mathrm{p}$.

18. 18. Fox Robin 1978, «Anthropologie de la parenté », Paris, Armand Colin.

19. Cécile Canut \& Etienne Smith (2006). « Pactes, alliances et plaisanteries », Cahiers d'études africaines, 184 /2006, consulté le 15 mars 2021.

20. De Marion Trévisi, M. (2008). «Au cour de la parenté : oncles et tantes dans la France des Lumières », books Google.com

21. Lefèbvre Jean Obélix (1985). « Nuit blanche », Le magazine du Livre, euridit.org.

22. Indépendant no 490 du vendredi 7 Février 2020.

23. www /http ; Wikipedia.org, consulté le 3/3/2020. 